\title{
EXTENSIONISMO EN LA CADENA PRODUCTIVA OVINOS EN LA CIUDAD DE MÉXICO
}

EXTENSIONISM IN THE SHEEP PRODUCTION CHAIN IN MEXICO CITY

\section{EXTENSIONISMO NA CADEIA PRODUTIVA DE OVINOS NA CIDADE DO MÉXICO}

Georgel Moctezuma López moctezuma.georgel@inifap.gob.mx INIFAP. Centro Nacional de Investigación Disciplinaria en Conservación y Mejoramiento de Ecosistemas Forestales

Ciudad de México - México

Eric Uriel Ramírez Sánchez extensionismo.cdmx@inifap.gob.mx UNAM - FES Aragón

Ciudad de México - México

Lourdes Velázquez Fragoso extensionismo.cdmx@inifap.gob.mx

Centro de Estudios Superiores - GAM

Ciudad de México - México

Diagramación Sindy Catherine Charcas Ibarra

Fotografia portada Santiago Ardila Acero

Encuentre este artículo en:

http://revistas.uniminuto.edu/index.php/IYD

Para citar este artículo / To cite this article

Moctezuma, G., Ramírez, E. \& Velázquez, L. (2021). Extensionismo en la cadena productiva ovinos en la Ciudad de México. Inclusión \& Desarrollo, 8 (1), pp 144-157

\section{RESUMEN}

Objetivo. Evaluar el programa de trabajo de los extensionistas pecuarios de la cadena productiva ovinos dirigido a pequeños productores dentro de los niveles I y II de la CDMX (aquellos que tiene las menores superficies y hatos ganaderos), con el acompañamiento del Instituto Nacional de Investigaciones Forestales, Agrícolas y Pecuarias (INIFAP), para presentar los resultados de las innovaciones tecnológicas que se implementaron en la cadena ovinos.
Fecha de recepción: 19 de junio de 2020 Fecha de aceptación: 10 de noviembre de 2020 Fecha de publicación: 1 de enero de 2021 
Metodología. Investigación cuantitativa basada en un análisis descriptivo de la evaluación de resultados obtenida a partir de la aplicación de varios indicadores a los datos obtenidos del instrumento de investigación denominado como bitácora (registro del trabajo) de campo, y cuya muestra se conformó de 81 ovinocultores los cuales representan al total de los productores atendidos por los extensionistas.

Resultados. Los logros que se obtuvieron por eslabón de la cadena productiva ovinos fueron los siguientes: para el caso de la producción se alcanzó el $94.4 \%$ de la meta, en transformación se obtuvieron los resultados más relevantes ya que se alcanzaron logros del $114.3 \%$ y $100 \%$, con respecto a la comercialización los resultados fueron los menos satisfactorios ya que se alcanzó $0 \%$ y $84.1 \%$ y con relación a la organización las metas se cubrieron en los niveles del $42.9 \%, 27.3 \%$ y $50 \%$.

Limitaciones. El programa de extensionismo funciona con presupuestos anuales, el cual tiene que ser autorizado para su renovación, lo cual implica un trámite burocrático que retrasa los inicios de las actividades y, por otro lado, tanto los extensionistas como los productores sujetos a atención no tienen continuidad, situación que no permite dar un seguimiento de mediano y largo plazo.

Originalidad. Las actividades de acompañamiento técnico - metodológico y asesoría se llevaron a cabo principalmente en doce comunidades con ovinocultores de escasos recursos de las cuatro alcaldías (antes delegaciones) del sureste de la Ciudad de México: Milpa Alta, Tláhuac, Tlalpan y Xochimilco.

Conclusión: Las acciones de asistencia técnica de los extensionistas pecuarios abarcaron cuatro eslabones de la cadena ovinos: i) producción, ii) transformación (industrialización o agregación de valor), iii) comercialización y iv) organización. Asimismo, los técnicos implementaron ocho innovaciones de la siguiente manera: eslabón primario, 1) medicina preventiva (inmunización y control parasitario) y 2) manejo del parto; eslabón transformación, 3) buenas prácticas de manufactura, 4) elaboración de productos cárnicos diferentes a la barbacoa y 5) elaboración de productos lácteos; eslabón de comercialización, 6) punto fijo de venta y 7) registros económicos para toma de decisiones y eslabón de organización, 8) asociación o agrupamiento de los productores de ovinos a partir de su capacidad productiva y la unificación de intereses.

Palabras clave: asistencia técnica, tecnología, evaluación, extensionismo e innovaciones.

\section{ABSTRACT}

Objetive. Evaluate the program of work of livestock extensionists of the sheep production chain aimed at small producers within levels I and II of the CDMX (those that have the smallest areas and livestock herds), with the accompaniment of the National Institute of Forestry, Agriculture and Livestock Research (INIFAP), to present the results of technological innovations that were implemented in the sheep chain.

Methodology. Quantitative research based on a descriptive analysis of the evaluation of results obtained from the application of various indicators to the data obtained from the research instrument known as the logbook (work record) field, and whose sample consisted of 81 ovinocultores which represent the total producers served by the extensionistas.

Results. The achievements that were obtained by link of the production chain sheep were the following: for the case of production $94.4 \%$ of the target was reached, in transformation the most relevant results were obtained since achievements of $114.3 \%$ and $100 \%$ were achieved, With respect to marketing, the results were the least satisfactory, as $0 \%$ and $84.1 \%$ were achieved, and with respect to the organization, the goals were met at the levels of $42.9 \%, 27.3 \%$ and $50 \%$.

Limitations. The extensionism program operates with annual budgets, which must be authorized for renewal, which implies a bureaucratic procedure that delays the start of activities and On the other hand, both the extensionists and the producers subject to care do not have continuity, a situation that does not allow for a medium and long-term follow-up.

Originality. The technical support - methodological and advisory activities were carried out mainly in twelve communities with low-income sheep farmers from the four municipalities (formerly delegations) of southeastern Mexico City: Milpa Alta, Tláhuac, Tlalpan and Xochimilco.

Conclusion: The technical assistance actions of the livestock extension workers covered four links of the sheep chain: i) production, ii) processing (industrialization or value aggregation), iii) marketing and iv)organization. Likewise, the

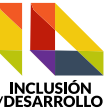


technicians implemented eight innovations as follows: primary link, 1) preventive medicine (immunization and parasite control) and 2) management of childbirth; transformation link, 3) good manufacturing practices, 4) manufacture of meat products other than barbecue and 5) manufacture of milk products; marketing link, 6) fixed point of sale and 7) economic records for decision-making and organisational link, 8) association or grouping of sheep producers on the basis of their productive capacity and the unification of interests.

Keywords: technical assistance, technology, evaluation, extensionism and innovations.

\section{SUMÀRIO}

Alvo. Avaliar o programa de trabalho dos extensionistas pecuários da cadeia produtiva de ovinos dirigido a pequenos produtores dentro dos níveis I e II da CDMX (aqueles que têm as menores superfícies e rebanhos de gado)com o acompanhamento do Instituto Nacional de Pesquisas Florestais, Agrícolas e Pecuárias (INIFAP), para apresentar os resultados das inovações tecnológicas que foram implementadas na cadeia de ovinos.

Metodologia. Investigação quantitativa baseada numa análise descritiva da avaliação dos resultados obtidos com a aplicação de vários indicadores aos dados obtidos a partir do instrumento de investigação denominado diário de bordo (registo do trabalho) de campo, cuja amostra foi constituída por 81 criadores de ovinos que representam o total dos produtores servidos pelos extensionistas.

Resultados. As realizações conseguidas por elo da cadeia produtiva dos ovinos foram as seguintes: para o caso da produção foi atingido $94.4 \%$ da meta, em transformação foram obtidos os resultados mais relevantes já que se alcançaram conquistas de $114.3 \%$ e $100 \%$, com respeito à comercialização os resultados foram os menos satisfatórios já que se alcançou $0 \%$ e $84.1 \%$ e com relação à organização as metas foram cobertas nos níveis de $42.9 \%, 27.3 \%$ e $50 \%$.

Limitações. O programa de extensionismo funciona com orçamentos anuais, que deve ser autorizado para a sua renovação, o que implica um trâmite burocrático que atrasa o início das atividades e, por outro lado, tanto os extensionistas como os produtores sujeitos a cuidados não têm continuidade, situação que não permite um acompanhamento de médio e longo prazo.

Originalidade. As atividades de acompanhamento técnico - metodológico e assessoria foram realizadas principalmente em doze comunidades com ovinocultores de escassos recursos das quatro prefeituras (anteriormente delegações) do sudeste da Cidade do México: Milpa Alta, Tláhuac, Tlalpan e Xochimilco.

Conclusão: As ações de assistência técnica dos extensionistas pecuários abrangeram quatro elos da cadeia ovina: i) produção, ii) transformação (industrialização ou agregação de valor), iii) comercialização e iv) organização. Além disso, os técnicos implementaram oito inovações da seguinte forma: elo primário, 1) medicina preventiva (imunização e controle parasitário) e 2) manejo do parto; elo transformação, 3) boas práticas de manufatura, 4) Fabrico de produtos à base de carne, com excepção dos churrascos, e 5) fabrico de produtos lácteos; elo de comercialização, 6) ponto fixo de venda e 7) registos económicos de tomada de decisão e elo de organização; 8) Associação ou agrupamento dos produtores de ovinos a partir da sua capacidade produtiva e da unificação de interesses.

Palavras chave: assistência técnica, tecnologia, avaliação, extensionismo e inovações. 


\section{Introducción}

En el proceso de PS\&E (planeación, seguimiento yevaluación), laactividad de evaluación, permite valorar de forma cuantitativa y cualitativa su importancia y es una herramienta básica para los directivos y tomadores de decisión que les permite continuar, modificar, redimensionar, posponer o bien cancelar los programas de desarrollo o de apoyos gubernamentales. Es conveniente recordar que evaluar, como lo señala Granger et al., 1995, es un juicio o una valoración del mérito, valor o calidad de la investigación, sea ella una propuesta, esté en desarrollo o haya sido concluida y por otro lado el Diccionario de la Real Academia de la Lengua Española (2017), menciona que es señalar el valor de algo, o bien, estimar, apreciar, calcular el valor de algo.

De acuerdo a la Secretaría de Agricultura y Desarrollo Rural, (SADER), dentro de su portal SERMEXICANO (2016) define al extensionismo como "el servicio prestado por personal de las instituciones de educación y de investigación que facilita el acceso al conocimiento, la información y las tecnologías, a productores, grupos y organizaciones económicas rurales y a otros actores del sector agropecuario, pesquero y acuícola". Con base a (Russo, 2009) el extensionismo es un proceso de comunicación que conlleva transferencia tecnológica a una población rural. Algunos autores se refieren al mismo como un vínculo dinámico entre la investigación científica y la producción agropecuaria (Engel, 2000). De acuerdo con el Consejo Mexicano para el Desarrollo Rural Sustentable (CMDRS, 2015) el extensionismo holístico abarca toda la cadena de valor, bajo una visión innovadora. La importancia del extensionismo pecuario en la Ciudad de México (CDMX) radica fundamentalmente en el enorme mercado de casi nueve millones de habitantes (CONAPO, 2018) que demandan una gran cantidad de productos alimenticios del sector ganadero. Así mismo (Reyes, 2013), señala que el resultado de la investigación agrícola en cualquier etapa de la agricultura debe ser puesto a disposición de los agricultores mediante la extensión agrícola, pues de otra manera pierde su valor. De lo anterior, se observa el compromiso del científico con el extensionista para vincularse sólidamente y llegar de una manera más efectiva y convincente a los productores del sector primario.

La Ciudad de México (CDMX) se ubica en el centro-sur de la república, territorialmente es el estado más pequeño, ya que tiene una superficie de $1,495 \mathrm{Km} 2$, lo que representa el $0.08 \%$ de la superficie total del país, su población es de 9.04 millones de habitantes (INEGI, 2016 y CONAPO, 2018), lo cual junto con la zona conurbana la convierten en una de las ciudades más pobladas del mundo y de igual manera, la participación económica de las actividades ganaderas (en las cuales se encuentra la ovinocultura) dentro del Producto Interno Bruto (PIB) de la CDMX es irrelevante ya que solo alcanzan niveles que van del $0.04 \%$ al $0.08 \%$ (INEGI,2015).

Las alcaldías de la CDMX en donde se realizan la mayor cantidad de actividades pecuarias en materia ovina son siete: Tlalpan, Milpa Alta, Xochimilco, Tláhuac, Álvaro Obregón, Cuajimalpa de Morelos y La Magdalena Contreras, sin embargo, en las tres primeras es donde se concentra la producción de borregos y en donde la suma de la superficie de las tres alcaldías que se mencionaron alcanza el $44.6 \%$ (Moctezuma, 2017 y 2018) de la superficie total de la CDMX. Con base a datos de la Comisión Técnico Consultiva de Coeficientes de Agostadero (COTECOCA) (2014), la entidad cuenta con 16,300 hectáreas de superficie ganadera, ocupada por agostaderos y praderas dedicadas principalmente a la cría de ganado en pastoreo. 
El programa extensionismo a cargo de la SADER en la CDMX y del gobierno capitalino durante el 2018 dirigió su atención principalmente a cinco cadenas de valor pecuarias, entre ellas la de ovinos. En la alcaldía de Tlalpan predomina la producción de ganado en pie y carne en canal de ovino y siguieron en orden de importancia las de Xochimilco y Milpa Alta. El hato ganadero de ovinos en 2017 (SIAP, 2017) en la CDMX fue de 10,154 cabezas, lo cual no es significativo dentro del inventario nacional ya que ni siquiera alcanza el $1 \%$ de la población ganadera nacional y su tendencia se observa en la Figura 1.

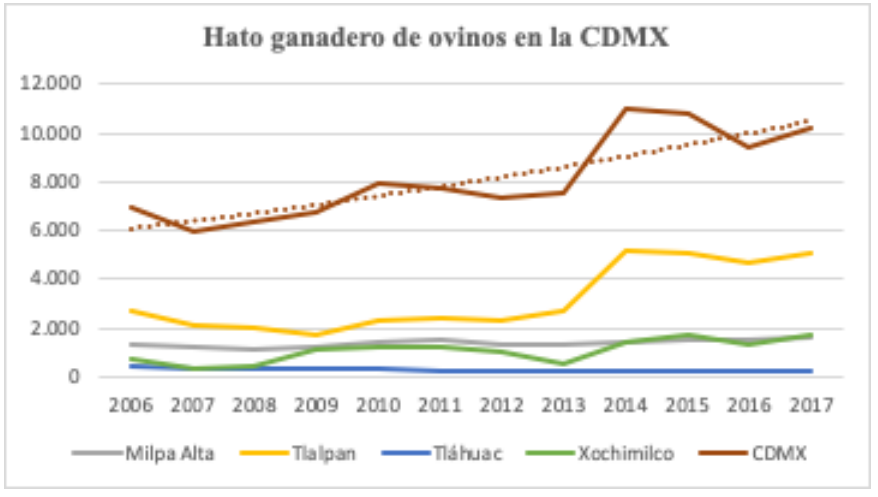

Figura 1. Hato ganadero de ovinos en la CDMX, durante el periodo $2006-2017$.

Fuente: SIACON, 2019

Con relación a la producción de carne y con datos de la misma fuente (SIAP, 2017) se obtuvieron en la CDMX 218.94 toneladas de carne, la tendencia durante el periodo 2006 2016 de la producción de carne de ovino es positiva con una tasa media de crecimiento del $3.29 \%$, a pesar de las cinco disminuciones en los años 2007, 2011, 2012, 2015 y 2016, las cuales coinciden con las disminuciones del hato de ovinos. La alcaldía más representativa durante todo el periodo es Tlalpan la cual muestra una tendencia parecida a la toda la CDMX (Figura 2).

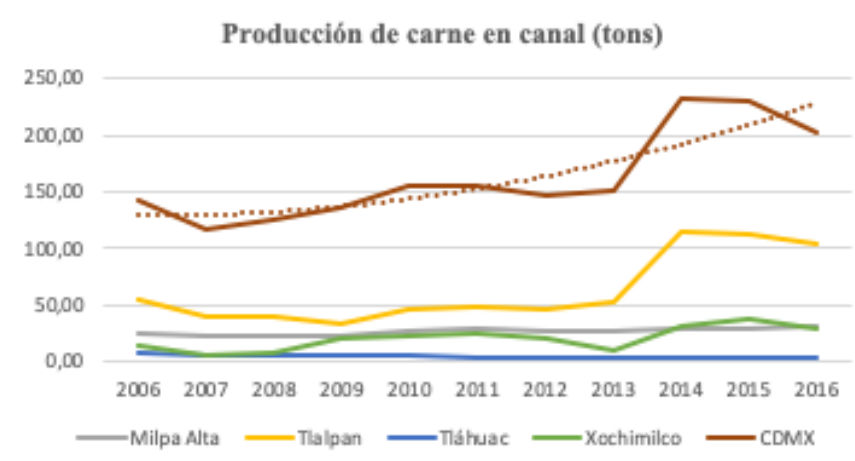

Figura 2. Producción de carne en canal de ovino en la CDMX, del periodo 2006 - 2016.

Fuente: SIACON, 2019

La anterior producción de carne en canal en la CDMX, al ser cuantificada en pesos, dejó un valor (a precios constantes de 2010) de \$ $13,395,000.00$ en 2016 , durante el periodo de 2006 a 2016, el valor de la producción muestra una tendencia favorable, con una pendiente positiva y una tasa de media de crecimiento anual del $5.6 \%$, la alcaldía de Tlalpan es la que mayor aporte económico genera con una tendencia muy similar a la de la CDMX, sin embargo, su tasa media de crecimiento anual es superior a la total, ya que alcanzó el $7.6 \%$ y supera a la tasa estatal en 2 puntos porcentuales (Figura 3).

Valor de la producción del canal de ovinos a precios constantes de $\mathbf{2 0 1 0}$ miles de \$

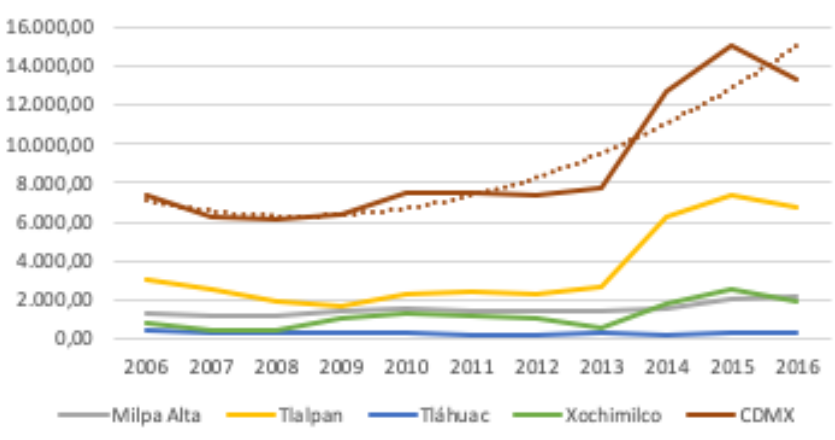

Figura 3. Valor de la producción de la carne en canal de ovino en la CDMX, durante el periodo $2006-2016$ a precios constantes con año base de 2010.

Fuente: SIACON, 2019 
El extensionismo en México es una práctica que se remonta desde la década de los 60's a través de la Fundación Rockefeller y se logró el establecimiento en la entonces Secretaría de Agricultura y Recursos Hidráulicos (SARH) la Dirección del Servicio de Extensión Agrícola con una cobertura de carácter nacional y sus técnicos eran contratados en forma directa por el Gobierno Federal y formaban parte de esa Secretaría de Estado. A finales de la década de los 80's el programa desaparece y en sustituido en 1995 por el Programa Alianza para el Campo en el cual uno de sus componentes fue el de extensionismo y de ese momento su nombre se modificó por ocho veces y pasó por manos de despachos privados, por asesores y posteriormente como pago por servicios profesionales de la Secretaría a los extensionistas. Esta situación provocó confusión dentro de los productores, así como de los propios técnicos (extensionistas) que no tenían continuidad y una incertidumbre dentro en su ingreso laboral. Como parte del acompañamientotecnológicodelasinstituciones de investigación y educación superior, se puso al alcance de los extensionistas los diversos paquetes tecnológicos generados y validados por esas entidades para que fuesen transferidos a los productores primarios, preferentemente de escasos recursos, y se implementaran las innovaciones acordes a sus características y actividades.

El objetivo del presente trabajo fue el de evaluar el programa de trabajo de los extensionistas pecuarios de la cadena productiva ovinos dirigido a pequeños productores dentro de los niveles I y II de la CDMX (aquellos que tiene las menores superficies y hatos ganaderos), con el acompañamiento del INIFAP, para advertir los resultados de las innovaciones implementadas en la cadena ovinos en el 2018.

\section{Metodología}

La presente investigación es de carácter cuantitativa basada en un análisis descriptivo sobre los resultados obtenidos de la instrumentación de las innovaciones tecnológicas en la cadena productiva ovinos, la evaluación de los resultados se realizó a partir de la aplicación de varios indicadores a los datos obtenidos del instrumento de investigación denominado como bitácora (registro del trabajo) de campo, los cuales se materializaron en las agendas de innovación y programas de trabajo de los extensionistas encargados de transferir las innovaciones tecnológicas a los ovinocultores; la muestra para realizar la investigación se conformó de 81 ovinocultores, lo que representa al total de los productores atendidos por los extensionistas.

El proceso metodológico que se utilizó para la evaluación de la cadena productiva ovinos como parte del componente del programa de extensionismo fue el de tomar en cuenta las acciones propuestas dentro del proyecto de Contribución Tecnológica del INIFAP al Extensionismo Rural de la anterior Secretaría de Agricultura, Ganadería, Desarrollo Rural, Pesca y Alimentación (SAGARPA) hoy Secretaría de Agricultura y Desarrollo Rural (SADER), en el cual el INIFAP tuvo como actividad principal la del acompañamiento tecnológico a los extensionistas que se contrataron en el programa, (Moctezuma, et al., 2017 y 2018) la cual consistió de las siguientes fases:

Selección de los extensionistas, se realizó por medio de una convocatoria de tipo abierta al público, por parte de la SAGARPA (hoy SADER) en la cual los aspirantes llenaron sus solicitudes y presentaron su examen de conocimientos en el portal de SERMEXICANO y posteriormente a los preseleccionados, se les llamó para que realizaran una entrevista presencial de aptitudes y actitudes y así elegir 
tanto a los extensionistas como al coordinador de los mismos (Vázquez y Landa, 2017).

Selección de cadenas de valor, con base al Plan Estratégico Agropecuario y Acuícola de la CDMX (2007), se seleccionaron seis cadenas pecuarias, entre ellas la de ovinos que es una de las que más aportan a la economía de la CDMX.

Elaboración de las agendas de innovación y programas de trabajo de los extensionistas a nivel individual y por cadena productiva, se solicitó a los extensionistas de manera individual y posteriormente de manera grupal sus programas de trabajo para de ahí derivar hacia la agenda de innovación de la cadena, la cual se basó fundamentalmente en la estrategia delineada por el Centro de Investigación en Estudios Sociales y Tecnológicos de la Agricultura yAgroindustria Mundial (CIESTAAM) de la Universidad Autónoma Chapingo (UACH), . (Manrubio et al., 2007) (Villareal 2015).

Socialización de las agendas de innovación, los extensionistas antes de iniciar sus labores de campo y de asistencia técnica realizaron reuniones de socialización con los ovinocultores para dar a conocer la agenda de innovación y que los productores emitieran sus comentarios y retroalimentaran a la agenda para la apropiación de la misma (Aguilar et al., 2010) (Saldaña et al., 2006).

Implementación de las innovaciones, se realizó una preselección de cuáles serían las innovaciones que tendría mayores posibilidades de éxito en su establecimiento, así como tuvieran la facilidad de que en el corto plazo se pudieran obtener resultados (Turrent 2017).

Recorridos de campo de supervisión, actividad que se realizó con la idea central de constatar y verificar en los ranchos y predios de los productores pecuarios la aplicación, adecuación o adopción de las innovaciones tecnológicas pecuarias en materia de ovinocultura; durante éstos recorridos se contó con la participación de los directivos de la SAGARPA (hoy SADER), del Instituto Nacional de Capacitación Rural (INCA Rural) así como del INIFAP.

Evaluación de la cadena productiva ovinos. para su valoración, se utilizó como punto de partida la línea base que se definió tanto en el programa de trabajo de los extensionistas de la cadena productiva ovinos como en la agenda de innovación 2018 y que tuvieran correspondencia con las metas que se establecieron y los resultados que lograron los productores con base a la asesoría técnica por parte de los extensionistas.

\section{Desarrollo y resultados}

Como parte de la selección de los profesionistas de la asistencia técnica, las cadenas productivas pecuarias de la CDMX más atendidas en cuanto a número de extensionistas, fue la cunícula con tres extensionistas, la de ovinos, apícola, avícola y porcinos con dos y la menos atendida fue la de bovinos leche con un solo extensionista y como parte del análisis se consideró a la coordinadora de extensionistas, Figura 4.

\footnotetext{
Número de extensionistas y su participación porcentual en las cadenas productivas pecuarias
}

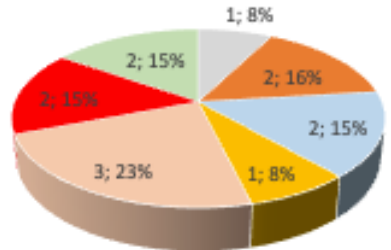

Cocrdinadora $=$ Apícola $=$ Avicola $=$ Bovinos leche $=$ Curicola - Ovinos $=$ Porcinos

Figura 4. Número de extensionistas y su participación porcentual en la atención a las cadenas productivas pecuarias durante 2018 en la CDMX

Elaboración propia con datos del componente extensionismo año 2018 
En el programa extensionismo pecuario en la CDMX, la participación de género es considerable ya que más de la mitad de los técnicos $(54 \%)$ son mujeres, dentro del cual destacan por sus aportaciones, colaboraciones yopiniones en el mejoramiento de las cadenas de valor pecuarias, así como en la implementación de las innovaciones tecnológicas propuestas por los extensionistas.

Con relación a los productores pecuarios de la cadena productiva ovinos, participantes en el proyecto de Apoyo al Extensionismo Rural, se solicitó a los extensionistas por parte de SAGARPA (SADER) y SEDERC, la conformación de un padrón de por lo menos 30 ovinocultores que estuvieran clasificados dentro de los niveles I y II de las reglas de operación del Componente Extensionismo para que se considerara su atención; con base a lo anterior el número de productores que se atendieron en la cadena ovinos se muestra en el Cuadro 1.

Cuadro 1. Padrón de productores pecuarios de ovinos en 2018, clasificados por género y cadena de valor que se atendieron en el componente extensionismo de la CDMX.

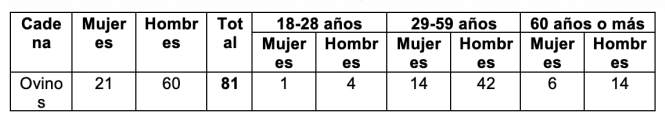

Elaboración propia con datos del componente extensionismo año 2018

La cadena productiva ovinos, atendió a un mayor número de productores, ya que la meta era de 60 y se contó con 81 ovinocultores, con lo cual la meta se superó en un $35 \%$. La mayor proporción de productores se concentró en la edad de 29 a 59 años lo que representó el $69.1 \%$ de la población que atendió la cadena y muestra que se encuentran en una edad totalmente productiva; el $24.7 \%$ del padrón están en una edad de plenitud y con una experiencia que puede ser aprovechada por los demás productores y únicamente el $6.2 \%$ de los borregueros son jóvenes, con lo cual se puede inferir que existe poco interés por esta actividad de carácter rural. La mujer participó con el $25.9 \%$ (poco más de la cuarta parte) de los participantes, lo cual demuestra en parte la feminización de la actividad ganadera ovina.

Las cuatro alcaldías y las 12 comunidades en las cuales se atendieron a los productores de ovinos fueron las siguientes:

Tlalpan: Parres El Guarda, San Miguel Topilejo, Santo Tomás Ajusco y San Miguel Xicalco.

Milpa Alta: San Lorenzo Tlacoyucan y San Pablo Oxtotepec.

Xochimilco: San Mateo Xalpa, San Francisco Tlanepantla y Santiago Tecalpatlalpan.

Tláhuac: San Pedro Tláhuac, San Francisco Tlaltenco y Santiago Zapotitlán.

En la parte de evaluación de innovaciones que se implementaron por parte de los productores se anotan lo que se realizó en cada uno de los eslabones de la cadena ovino que se programaron.

Eslabón primario; el indicador que se utilizó fue el de unidades de producción con una mortalidad $\leq$ a 10 , en el cual la línea base fue de 12 y la meta propuesta fue de 18 . El resultado que se dio fue de 17 , con lo cual el logro que se alcanzó fue del $94.4 \%$.

Para el logro anterior se implementaron dos innovaciones de acuerdo al Cuadro 2. 
Cuadro 2. Innovaciones en el eslabón primario implementadas y acciones o actividades para su éxito.

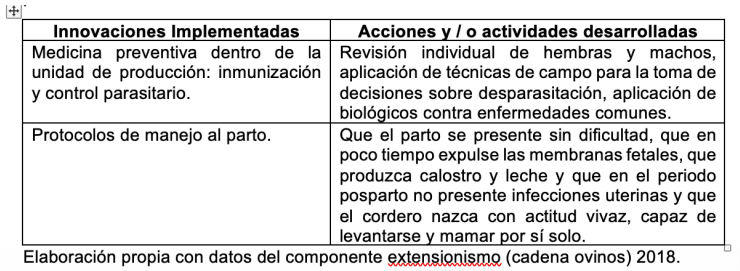

Eslabón de transformación, los indicadores que se utilizaron fueron dos: productores transformando cárnicos y productores transformando lácteos. Las líneas base para cada uno de los indicadores fueros de 5 productores para el primer caso y de 0 para el segundo y las metas que se propusieron fueron de 7 y 2 productores para el caso de cárnicos y lácteos respectivamente. Los resultados que se dieron fueron de 8 y 2 , con lo cual el logro que se alcanzó en los productores de cárnicos fue del $114.3 \%$ y de los lácteos fue del $100 \%$

Para alcanzar los resultados en el eslabón de transformación se implementaron tres innovaciones de acuerdo al Cuadro 3.

Cuadro 3. Innovaciones en el eslabón de transformación implementadas y acciones 0 actividades para su éxito.

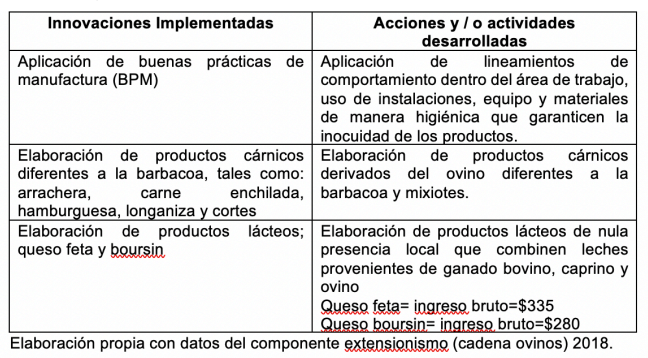

Eslabón de comercialización, los indicadores que se utilizaron fueron dos: puntos de venta fijo e ingresos brutos obtenidos por productos comestibles con valor agregado por animal. Las líneas base para cada uno de los indicadores fueros de 0 puntos de venta fijos y $\$ 0.00$ en cada uno de los indicadores y las metas que se propusieron fueron de 1 punto de venta fijo y $\$$ $1,725.00$ de ingresos brutos respectivamente. Los resultados que se dieron fueron de 0 en el punto fijo de venta y de $\$ 1,450.00$ de ingreso bruto por animal, con lo cual el logro que se alcanzó en el punto de venta fijo fue nulo y en el caso de los ingresos brutos sólo se alcanzó el $84.1 \%$

Para alcanzar los resultados se implementaron en el eslabón de comercialización, dos innovaciones de acuerdo al Cuadro 4.

Cuadro 4. Innovaciones en el eslabón comercialización implementadas y acciones o actividades para su éxito.

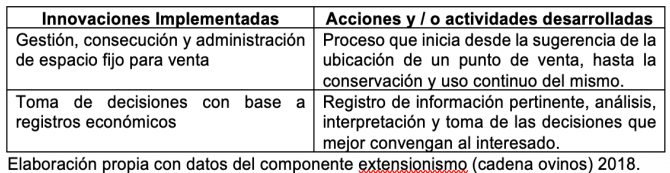

Elaboración propia con datos del componente extensionismo (cadena ovinos) 2018.

Eslabón de organización, los indicadores que se utilizaron fueron tres: productores que colaboran para transformar, productores que colaboran para comercializar y grupos organizados. Las líneas base para cada uno de los indicadores fueros de 5 productores que transforma, 9 productores que comercializan y 1 grupo organizado en cada uno de los indicadores y las metas que se propusieron fueron de 7 productores transformadores, 11 productores comercializadores y 2 grupos organizados respectivamente. Los resultados que se dieron fueron de 3 productores en la transformación, 3 productores en la comercialización y 1 grupo organizado, con lo cual los logros que se alcanzaron fueron del $42.9 \%$ para los productores que colaboran en la transformación, $27.3 \%$ en los productores que colaboran en la comercialización y del $50 \%$ 
para los grupos organizados.

Para alcanzar los resultados se implementó en el eslabón de organización, una innovación de acuerdo al Cuadro 5.

Cuadro 5. Innovaciones en el eslabón de organización implementadas y acciones 0 actividades para su éxito.

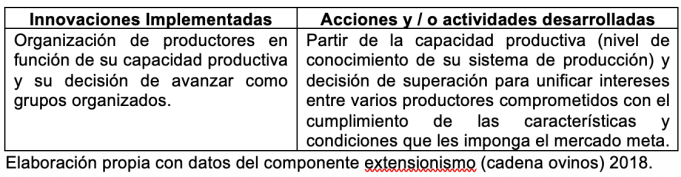

Elaboración propia con datos del componente extensionismo (cadena ovinos) 2018.

Los beneficios que obtuvieron los ovinocultores fueron: incremento y mejora en la salud animal, menor mortalidad, transformación de productos pecuarios para dar valor agregado, mayor diversidad de productos y calidad de productos finales al mercado, incremento en ingreso y mayor conciencia en la trascendencia de la organización de productores.

A manera de resumen se presentan los resultados de los eslabones de la cadena productiva ovinos que se atendió mediante asistencia técnica por parte de los extensionistas de la CDMX y que se muestran en el Cuadro 6.

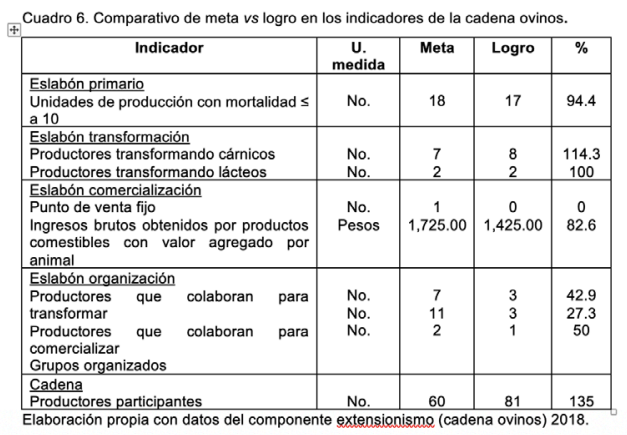

El mayor logro dentro de la cadena productiva ovino fue el relativo a número de productores participantes y en cuanto a los eslabones, el de transformación alcanzó los mejores porcentajes y los eslabones de comercialización y organización no alcanzaron sus metas en mayor proporción y al primario le faltó un porcentaje pequeño para alcanzar la meta.

\section{Conclusiones}

El programa extensionismo en materia de ovinocultura en la CDMX se desarrolló en las alcaldías: Tlalpan, Milpa Alta, Tláhuac y Xochimilco, en la primera es dónde se concentra la mayor producción de borregos con más de la mitad (51.2\%), en la misma proporción en lo relativo a la importancia económica, el mayor hato ganadero de ovinos se encuentra también en la alcaldía de Tlalpan y tiene una tendencia positiva en cuanto a su crecimiento.

La meta de atención a productores de ovinos se superó en un 35\% y se observó una participación del $25.9 \%$ por parte de las mujeres lo que indica la feminización del campo mexicano. La participación de productores jóvenes en la cadena ovinos de la CDMX es escasa ya que sólo alcanzó un $6.2 \%$

Las actividades de los extensionistas abarcaron cuatro eslabones de la cadena: producción, transformación, comercialización y organización. Además, implementaron ocho innovaciones de la siguiente manera: eslabón primario con medicina preventiva (inmunización y control parasitario) y manejo del parto; eslabón transformación con buenas prácticas de manufactura (BPM), elaboración de productos cárnicos diferentes a la barbacoa y elaboración de productos lácteos; eslabón de comercialización con un punto fijo de venta y registros económicos para toma de decisiones y eslabón de organización mediante su agrupamiento (o asociación) a partir de su capacidad productiva y la unificación de intereses. 
Las innovaciones implementadas por los productores de ovinos bajo la supervisión de los extensionistas fueron: técnicas de alimentación, manejo de abonos, implementación de buenas prácticas de producción, uso de medicamentos, control de plagas y enfermedades, elaboración de productos cárnicos, fabricación de cosméticos, curtido de piel, manejo e implementación de reproducción intensiva.

Los logros que se obtuvieron por eslabón de la cadena fueron los siguientes: para el caso de producción se alcanzó el $94.4 \%$ de la meta, en transformación se obtuvieron los resultados más relevantes ya que se alcanzaron logros del $114.3 \%$ y $100 \%$, con relación a la comercialización los resultados fueron los menos satisfactorios ya que se alcanzó $0 \%$ y $84.1 \%$ y con relación a la organización las metas se cubrieron en los niveles del $42.9 \%$, $27.3 \%$ y $50 \%$

Elmayorbeneficioquedetectaronlosproductores pecuarios de ovinos fue el de agregación de valor por medio de la transformación de productos cárnicos diferentes al tradicional de la barbacoa, lo cual les permitió incrementar su ingreso y finalmente. 


\section{Referencias bibliográficas}

Aguilar, A. J., Santoyo, J. L., Solleiro, J. R., Altamirano, C. \& Baca, J. (2005). Transferencia e Innovación Tecnológica en la Agricultura. Lecciones y Propuestas. Fundación Produce Michoacán. México: Universidad Autónoma Chapingo.

Aguilar, A.J., Altamirano, J. R. \& Rendón, M. R. (2010). Del Extensionismo Agrícola a las Redes de Innovación Rural. México: Universidad Autónoma Chapingo. Recuperado de: http:// www.redinnovagro.in/documentosinnov/extensionismo_30_sept.pdf

Comisión Nacional de Población. (2015). Anuarios Estadísticos. México: CONAPO.

Comisión Nacional de Población. (2018). Indicadores Demográficos Ciudad de México 2018. Recuperado de http://www.conapo.gob.mx/work/models/CONAPO/Mapa_Ind_Dem18/index.html

Comisión Técnico Consultiva de Coeficientes de Agostadero. (2014). Superficie Ganadera. Recuperado de: http://apps1.semarnat.gob.mx/dgeia/compendio_2016/archivos/02_agrigan/D2_AGRIGAN04_02.pdf consultada en febrero de 2019

Consejo Mexicano para el Desarrollo Rural Sustentable. (2015). Desarrollo de Capacidades y Extensionismo Rural. Recuperado de http://www.cmdrs.gob.mx/sesiones/Documents/2015/2a_sesion/4_extencionismo.pdf

Engel, P. (2000). Facilitando el desarrollo sostenible: ¿hacia una extensión moderna? Centro de Estudios y Gestión para el Desarrollo Rural Sostenible (CEDRO). Chile: Universidad de Concepción.

Gobierno del Distrito Federal. (2009). Diagnóstico del Sector Rural en el Distrito Federal. Recuperado de: http://www.sagarpa.gob.mx/Delegaciones/distritofederal/Documents/Evaluaciones/2008-2010\%20Diagnostico\%20Sector\%20Rural.pdf

Granger, A., Grierson, J., Quirino, T. R. \& Romano, L. (1995). Evaluación en la Administración de la Investigación Agropecuaria. Servicio Internacional para la Investigación Agrícola Nacional (ISNAR), Módulo 4 de la serie de Capacitación en Planificación, Seguimiento y Evaluación para la Administración de la Investigación Agropecuaria. Colombia: ISNAR.

Timothy, G. \& Valentine, H. (2008). Sampling Strategies for Natural Resources and Environment. Reino Unido: Chapman and Hall / CRC. Recuperado de: https://www.crcpress.com/ Sampling-Startegies-for-Natural-Resources-and-the-Environment/Gregoire-Valentine/p/ book/9781584883708

Instituto Nacional de Estadística y Geografía. (2007). Censo Agrícola, Ganadero y Forestal 2007. México: INEGI

Instituto Nacional de Estadística y Geografía. (2015). PIB- Entidad Federativa, anual. Recuperado de: http://www.inegi.org.mx/est/contenidos/proyectos/cn/pibe/tabulados.aspx Instituto Nacional de Estadística y Geografía. (2016). México en Cifras. Recuperado de: https://www.inegi.org.mx/app/areasgeograficas/?ag=09

Muñoz, M., Altamirano, J., Aguilar, J., Rendón, R. \& Espejel, A. (2007). Innovación: Motor de la Competitividad Agroalimentaria - Políticas y Estrategias para que en México Ocurra -. México: Universidad Autónoma Chapingo. Recuperado de: https://ciestaam.edu.mx/libro/ 
innovacion-motor-la-competitividad-agroalimentaria-politicas-estrategias-en-mexico-ocurra/

Moctezuma, G., Espinosa, J., Romero, M., Castillo, A. \& Castillo, R. (2017). Evaluación del Componente Extensionismo Pecuario en la Ciudad de México (CDMX). Memoria II Seminario Nacional de Investigación Socioeconómica y Ambiental de la Producción Pecuaria. Colegio de Postgraduados. Puebla, Pue. México. Colegio de Postgraduados.

Moctezuma, G., Ramírez, E., Velázquez, L., Vélez, A. \& Romero, M. (2018). Extensionismo rural en la Ciudad de México: aportes del INIFAP al desarrollo de capacidades en el campo. Memorias 60 Años de Investigación Forestal en Coyoacán. INIFAP. CENID - COMEF. Ciudad de México. México: INIFAP.

Real Academia de la Lengua Española. (2019). Diccionario de la Real Academia de la Lengua Española. España: RAE.

Reyes, S. (2013). El Servicio de Extensión Rural en México. Propuestas de Política Pública. México: Colegio de Postgraduados.

Saldaña, R., Espinosa, J., Moctezuma, G. Ayala, A., Tapia, C., Ríos, R., Valle, S. \& Gomes, A. (2006). Proyecto Quo Vadis: El Futuro de la Investigación Agropecuaria y Forestal y la Innovación Institucional de México. México: INIFAP.

Russo, O. (2009). Capacidades y Competencias del Extensionista. Agropecuario y Forestal en la Globalización. Comunicación. Costa Rica: Instituto Tecnológico de Costa Rica.

Secretaría de Agricultura, Ganadería Desarrollo Rural, Pesca y Alimentación. (2007). Programa Nacional Pecuario. México: SAGARPA.

Secretaría de Agricultura, Ganadería Desarrollo Rural, Pesca y Alimentación. (2016). SER MEXICANO. Recuperado de: http://www.extensionismo.mx/web1/index.php/contenido/119-extensionismo-rural

Servicio de Información Agroalimentaria y Pesquera. (2015). Población ganadera 20062015. Recuperado de: http://www.gob.mx/cms/uploads/attachment/file/165998/bovlech.pdf consultada en febrero de 2019

Servicio de Información Agroalimentaria y Pesquera. (2016). Ganado en pie y carne de en canal por municipio y año. Recuperado de: http://infosiap.siap.gob.mx/annpecuaio_siapx_ gobmx/apecmpio.jsp?id=3consultada en febrero de 2019

Servicio de Información Agroalimentaria y Pesquera. (2017). Población ganadera 20062015. Recuperado de: http://www.gob.mx/cms/uploads/attachment/file/165998/bovlech.pdf consultada en febrero de 2019.

Turrent, A. (2007). Políticas de Investigación y Transferencia Agrícola, Pecuaria y Forestal para el Campo Mexicano. En Calva, J. (Coord.). Desarrollo Agropecuario y Forestal. Agenda para el Desarrollo. Volumen 9. México: Editorial Miguel Ángel Porrúa.

Vázquez, R. \& Landa, E. (2017). Relación entre el perfil técnico de los extensionistas pecuarios y su desempeño. Memoria. Reunión Nacional de Investigación Pecuaria. Ciencia y Tecnología para la Ganadería Tropical Mexicana. México: INIFAP 
Villareal, R. (2015). Extensionismo Holístico y el Agrocluster. Modelo de Asociatividad e Integración de la Cadena Productiva. México: SAGARPA.

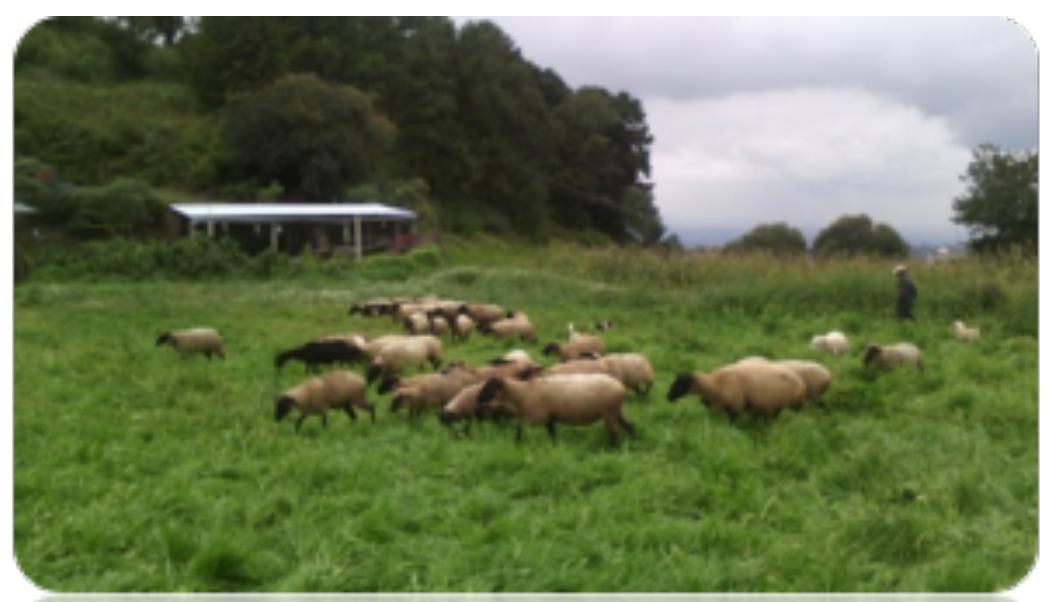

Hato de ovinos en pastoreo característico de la CDMX. 